\title{
Lung cancer screening in never-smokers: facts and remaining issues
}

\author{
Maurizio V. Infante ${ }^{1}$ and Giuseppe Cardillo ${ }^{2}$ \\ Affiliations: ${ }^{1}$ Thoracic Surgery Dept, University and Hospital Trust - Borgo Trento, Verona, Italy. ${ }^{2}$ Thoracic \\ Surgery Unit, Azienda Ospedaliera San Camillo Forlanini, Rome, Italy. \\ Correspondence: Maurizio V. Infante, Thoracic Surgery Dept, University and Hospital Trust - Borgo Trento, \\ P.le A. Stefani, 1 - 37126 Verona, Italy. E-mail: maurizio.infantedaoovr.veneto.it
}

@ERSpublications

A similar probability of unwanted harm is to be expected in both ever- and never-smokers undergoing CT screening, together with a slightly lower lung cancer detection rate in the latter https://bit.ly/3izc1ZW

Cite this article as: Infante MV, Cardillo G. Lung cancer screening in never-smokers: facts and remaining issues. Eur Respir J 2020; 56: 2002949 [https://doi.org/10.1183/13993003.02949-2020].

The final publication of the NELSON trial data [1] has corroborated the findings of the National Lung Screening Trial (NLST) confirming the ability of lung cancer screening with low-dose computed tomography (LDCT) to detect lung cancer at an early stage and significantly reduce lung cancer mortality in high-risk groups [2].

Since then, the European Respiratory Society and the European Society for Radiology have agreed that Europe's healthcare systems should allow citizens at risk to benefit from organised pathways to early diagnosis of lung cancer [3] and efforts are ongoing to obtain governmental approval for publicly funded screening programmes in European countries, as has already happened in the USA.

However, the mortality benefit of LDCT screening was only demonstrated in heavy smokers in an age range above 50 or 55 years, and in Western countries, where lung cancer in never-smokers is 12-30 times less frequent than in smokers [4].

Unfortunately, lung cancer in never-smokers has been steadily increasing worldwide in the past few decades and it now ranks seventh among the causes of cancer death [5-7]. It has a particularly high impact in Asia, where it peaks as high as $39.7 \%$ of all lung cancer patients in China, and at $38 \%$ in South Korea, compared to only $10 \%-15 \%$ in Europe and North America [8].

Irrespective of geographical area, female patients are more prevalent, but particularly so in Asian countries where up to $83 \%$ of female lung cancer patients are never-smokers [5].

A number of factors have been implicated in the pathogenesis of lung cancer in never-smokers, such as exposure to second-hand smoke, the use of solid fuels and indoor cooking in poorly ventilated households (mainly in low-income countries), particulate matter inhalation, and radon gas [9, 10], while familial associations strongly suggest the importance of genetic predisposition $[11,12]$.

It has become increasingly clear that lung cancer in never-smokers has different biological features compared with ever-smokers [13]: peripheral adenocarcinoma is largely prevalent in never-smokers, most frequently of the types now classified as minimally invasive adenocarcinoma (MIA) or lepidic-predominant adenocarcinoma (formerly bronchioloalveolar carcinoma), that have their radiological counterparts in ground-glass nodules [14]. 
On the contrary squamous cell carcinomas are poorly represented in never-smokers, and small cell carcinomas are virtually absent. Smokers too can develop adenocarcinoma of course, but in them it will present more often as a solid nodule on CT and tends to have a more aggressive behaviour.

Genetic alterations also differ in lung cancers of never-smokers.

Epidermal growth factor receptor (EGFR) mutations are the most common oncogenic driver in never-smoker pulmonary adenocarcinoma in East Asia (60-78\% of this subgroup), while they can be found only in about $10-15 \%$ in western countries, and the frequency of EGFR-mutated lung cancer decreases with increasing smoking exposure. Other oncogenic drivers may be predominantly identified in never-smokers with adenocarcinoma, such as ROS1 rearrangement, but can also been found in patients with other histotypes.

On the other hand, KRAS mutations pertain almost exclusively to lung cancer in smokers, and P53 mutations, which can be observed both in patients who have never smoked and in those with tobacco-associated lung cancer, appear with different frequency and show different mutational signatures and spectra $[7,13,15,16]$.

Finally, pathological features of aggressive lung cancer are much less frequent in never-smokers who have better cancer-specific and overall survival after treatment regardless of stage $[17,18]$.

Targeting the appropriate population for a LDCT screening programme is of paramount importance in order to maximise the potential benefits of screening and minimise unwanted harm and added costs of false positive findings, overtreatment of indolent lesions, and increased use of primary and secondary care resources by screened subjects [19].

A number of risk models have been developed in an attempt to detect as many lung cancer patients as possible in asymptomatic high-risk groups and to keep as many non-lung cancer patients as possible away from harm.

With one exception [20] in Western countries current risk models including the most widely adopted PLCOm2012 do not include never-smokers [21].

However, some data $[22,23]$ suggest that the impact of smoking may be relatively less important in East Asia than in Europe and North America.

By applying a risk model that includes sex, age, family history and analysis of a set of single-nucleotide polymorphisms that were found to be associated with lung cancer risk, YANG [24] reported a high 2.34\% lung cancer detection rate in a screening trial that specifically targets never-smokers in Taiwan.

Prospective trials of LDCT screening started in Japan in the late-1990s that included never-smokers and subjects as young as 40 years [23], and many observational studies or voluntary paid CT screening programmes have been active since then in Asian countries (table 1).

TABLE 1 Prevalence of BAC-AAH-AIS-MIA in CT screening programmes in Asian countries and in the NLST

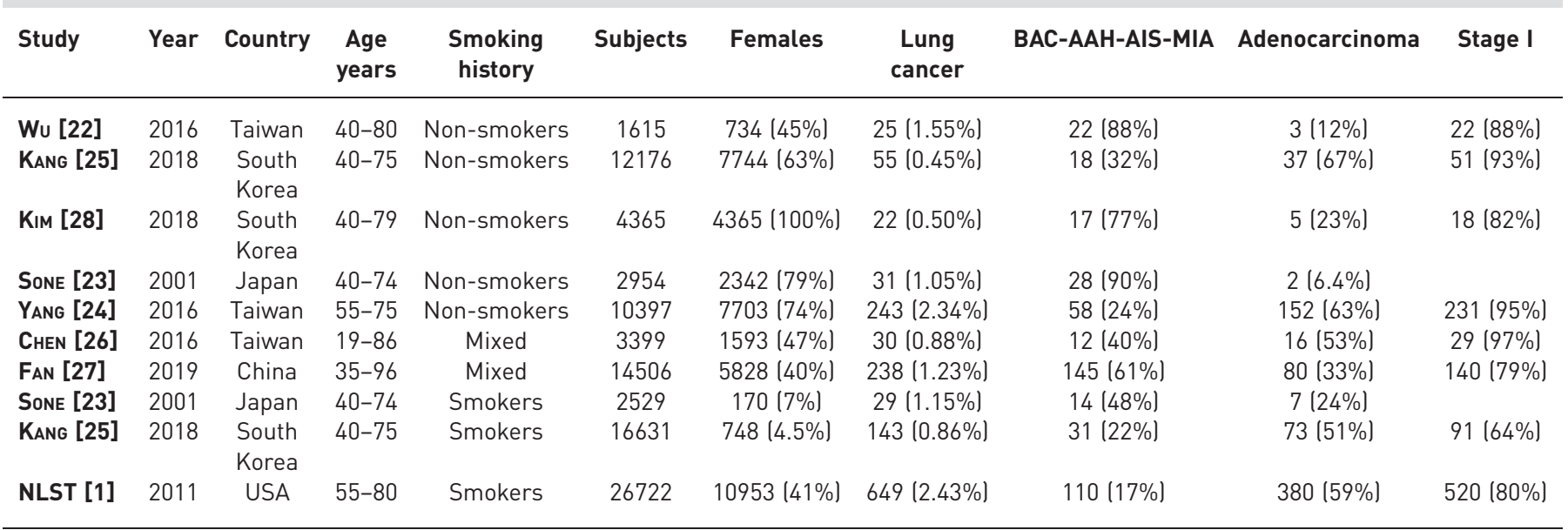

BAC: bronchioloalveolar carcinoma; AAH: atypical adenomatous hyperplasia; AIS: adenocarcinoma in situ; MIA: minimally invasive adenocarcinoma. These entities are here presented as one group. 
Data on lung cancer overdiagnosis of inconsequential, indolent lung tumours (pseudodisease) with LDCT screening in randomised LDCT trials vary widely. In the NLST it is estimated at $18 \%$ [29], while in the Danish Lung Cancer Screening trial at 60\% [30].

Overdiagnosis of indolent disease and some unwanted harm are the price to pay in any screening programme and are hardly avoidable; for a population screening programme to be effective and worthwhile however, overdiagnosis and harm must be compensated by a reduced mortality rate in the screened population.

In other words, the goal of lung cancer screening should be a reduction in lung cancer mortality and not simply an increase in the detection of "early-stage" indolent tumours.

In European and North American screening trials, the observed prevalence of tumours manifesting as ground-glass nodules (GGNs) is quite low (4-16\%) [31-33], a striking difference with screening trials in Asian countries and, in particular, in Asian female never-smokers, where a very high prevalence of subsolid lesions is reported (table 1).

There is ample observational evidence that many of these are fairly unaggressive and may at least in part represent overdiagnosis.

In Europe, SiLva et al. [33] and Scholten et al. [34] have reported on the outcomes of persistent subsolid nodules detected by LDCT screening and followed for prolonged periods. In both studies, the risk of dying of a lung cancer developing from a subsolid nodule was exceedingly low and long-term observation of such lesions was advocated.

KAKINUMA et al. [35] reported on long-term observation of subjects with persistent GGNs in Japan. While part-solid nodules (with a solid component measuring up to $5 \mathrm{~mm}$ in a mediastinal window) had a $48 \%$ probability of growth within 5 years, the probability of growth by at least $2 \mathrm{~mm}$ at 5 years was only $12-$ $24 \%$ in pure or "heterogeneous" GGNs.

KоваYASH et al. [36] recently reviewed recent reports on patients with GGNs whose smoking history was available, and observed that all data consistently showed that persistent GGNs in never-smokers were significantly less likely to grow, and less likely to increase in number than in smokers.

A clue that the risk of overdiagnosis in never-smokers undergoing LDCT screening in Asia might be relevant is offered by Kondo et al. [37], who reported a high incidence of non-solid nodules in non-smokers who underwent LDCT screening; in this subgroup, there was a low resection rate of $45 \%$ due to continued observation in many such cases. Despite this, the mortality rate due to lung cancer was only $3.7 \%$ in this group, while a $79 \%$ resection rate and $29 \%$ mortality rate was observed in lung cancers detected by chest radiograph, most of which were solid lesions.

In China, LiANG et al. [38] have recently observed that lung cancer incidence in females in the outskirts of Shanghai have risen significantly since LDCT screening was introduced but lung cancer mortality rate remains stable: a hallmark of overdiagnosis.

On the other hand, although such tumours tend to have an indolent course and may not grow for many years, we have little data about the fate of younger patients (e.g. in their forties) who have been diagnosed with subsolid nodules and followed lifelong.

Clearly, some GGNs do have a potential to progress and become truly malignant, albeit after many years [39]; but there would be plenty of time for them to grow when they first appear in relatively young patients.

In this current issue of the European Respiratory Journal, KIM et al. [40] present data from a huge retrospective cohort study of 37436 asymptomatic adults who underwent LDCT screening in South Korea, $48 \%$ of whom were never-smokers.

A detailed description of abnormal findings, false positives and invasive procedures is provided in both groups, showing that a similar probability of unwanted harm is to be expected in both ever- and never-smokers undergoing CT-screening, together with a slightly lower lung cancer detection rate in the latter.

Strong correlations between age and lung cancer risk were observed in never-smokers by KANG et al. [25] in a previous work on the same patient population, where lung cancer detection rate was only $0.16 \%$ in patients below the age of 50 years.

Another group recently reported on 4365 female never-smokers aged 40-79 years enrolled in another lung cancer screening programme in Korea [28]. Lung cancer detection rate was $0.5 \%$ overall, but female never-smokers who had Lung-RADS category 1-3 findings at baseline had only $0.07 \%$ probability of developing subsequent lung cancer at 5 years and only $0.17 \%$ at 10 years. 
Current data suggests therefore that sex, age, family history, and the results of the baseline CT-scan if already available should be taken into account when considering lung cancer CT screening for never-smokers. It is reasonable to assume that the balance between benefits of CT screening and its downsides will not be the same for everyone.

Potential benefits of screening for lung cancer in terms of mortality reduction should outweigh potential harm and financial burden, also in never-smokers. These metrics should be assessed in a controlled fashion in large enough randomised trials, but such data is not available as yet and is not going to become available anytime soon.

Despite such a knowledge gap, lung cancer screening in never-smokers is already commonplace and it will certainly continue to expand in the near future at least in Asia, so the more information is available, the better doctors can do for their patients.

The information provided by KIM et al. [40] on lung cancer detection rate, false positives and on the probability of facing unwanted consequences can be useful for individual patient counselling on a decision that can have an important impact on their health.

Conflict of interest: M.V. Infante has nothing to disclose. G. Cardillo has nothing to disclose.

\section{References}

1 Aberle DR, Adams AM, Berg CD, et al. National Lung Screening Trial Research Team. Reduced lung-cancer mortality with low-dose computed tomographic screening. N Engl J Med 2011; 365: 395-409.

2 de Koning HJ, van der Aalst CM, de Jong PA, et al. Reduced lung-cancer mortality with volume CT screening in a randomized trial. $N$ Engl J Med 2020; 382: 503-513.

3 Kauczor HU, Baird AM, Blum TG, et al. ESR/ERS statement paper on lung cancer screening. Eur Radiol 2020; 30: 3277-3294.

4 Pirie K, Peto R, Green J, et al. Lung cancer in never smokers in the UK Million Women Study. Int J Cancer 2016; 139: 347-354.

5 Barta JA, Powell CA, Wisnivesky JP. Global epidemiology of lung cancer. Ann Glob Health 2019; 85: 8.

6 Wakelee HA, Chang ET, Gomez SL, et al. Lung cancer incidence in never smokers. J Clin Oncol 2007; 25: 472-478.

7 Pallis AG, Syrigos KN. Lung cancer in never smokers: disease characteristics and risk factors. Crit Rev Oncol Hematol 2013; 88: 494-503.

8 McCarthy WJ, Meza R, Jeon J, et al. Chapter 6: Lung cancer in never smokers: epidemiology and risk prediction models. Risk Anal 2012; 32: Suppl 1, S69-S84.

9 Gordon SB, Bruce NG, Grigg J, et al. Respiratory risks from household air pollution in low and middle income countries. Lancet Respir Med 2014; 2: 823-860.

10 Kim AS, Ko HJ, Kwon JH, et al. Exposure to secondhand smoke and risk of cancer in never smokers: a meta-analysis of epidemiologic studies. Int J Environ Res Public Health 2018; 15: 1981.

11 Carr SR, Akerley W, Hashibe M, et al. Evidence for a genetical contribution to non-smoking-related lung cancer. Thorax 2015; 70: 1033-1039.

12 Lin KF, Wu HF, Huang WC, et al. Propensity score analysis of lung cancer risk in a population with high prevalence of non-smoking related lung cancer. BMC Pulm Med 2017; 17: 120.

13 Sun S, Schiller JH, Gazdar AF. Lung cancer in never smokers - a different disease. Nat Rev Cancer 2007; 7: 778-790.

14 Travis WD, Brambilla E, Noguchi M, et al. International Association for the Study of Lung Cancer/American Thoracic Society/European Respiratory Society international multidisciplinary classification of lung adenocarcinoma. J Thorac Oncol 2011; 6: 244-285.

15 Subramanian J, Govindan R. Molecular genetics of lung cancer in people who have never smoked. Lancet Oncol 2008; 9: 676-682.

16 Sun Y, Ren Y, Fang Z, et al. Lung adenocarcinoma from East Asian never-smokers is a disease largely defined by targetable oncogenic mutant kinases. J Clin Oncol 2010; 28: 4616-4620.

17 Yano T, Miura N, Takenaka $\mathrm{T}$, et al. Never-smoking nonsmall cell lung cancer as a separate entity: clinicopathologic features and survival. Cancer 2008; 113: 1012-1018.

18 Janjigian YY, McDonnell K, Kris MG, et al. Pack-years of cigarette smoking as a prognostic factor in patients with stage IIIB/IV nonsmall cell lung cancer. Cancer 2010; 116: 670-675.

19 Jensen MD, Siersma V, Rasmussen JF, et al. Direct and indirect healthcare costs of lung cancer CT screening in Denmark: a registry study. BMJ Open 2020; 10: e031768.

20 Cassidy A, Myles JP, van Tongeren M, et al. The LLP risk model: an individual risk prediction model for lung cancer. Br J Cancer 2008; 98: 270-276.

21 Tammemägi MC, Church TR, Hocking WG, et al. Evaluation of the lung cancer risks at which to screen everand never-smokers: screening rules applied to the PLCO and NLST cohorts. PLoS Med 2014; 11: e1001764.

22 Wu FZ, Huang YL, Wu CC, et al. Assessment of selection criteria for low-dose lung screening CT among Asian ethnic groups in Taiwan: from mass screening to specific risk-based screening for non-smoker lung cancer. Clin Lung Cancer 2016; 17: e45-e56.

23 Sone S, Li F, Yang ZG, et al. Results of three-year mass screening programme for lung cancer using mobile low-dose spiral computed tomography scanner. Br J Cancer 2001; 84: 25-32.

24 Yang P. MS16.04 National lung screening program in Taiwan. J Thorac Oncol 2018; 13: S274-S275. 
25 Kang HR, Cho JY, Lee SH, et al. Role of low-dose computerized tomography in lung cancer screening among never-smokers. $J$ Thorac Oncol 2019; 14: 436-444.

26 Chen $\mathrm{CY}$, Chen $\mathrm{CH}$, Shen TC, et al. Lung cancer screening with low-dose computed tomography: experiences from a tertiary hospital in Taiwan. J Formos Med Assoc 2016; 115: 163-170.

27 Fan L, Wang Y, Zhou Y, et al. Lung cancer screening with low-dose CT: baseline screening results in Shanghai. Acad Radiol 2019; 26: 1283-1291.

28 Kim HY, Jung KW, Lim KY, et al. Lung cancer screening with low-dose CT in female never smokers: retrospective cohort study with long-term national data follow-up. Cancer Res Treat 2018; 50: 748-756.

29 Patz EF Jr, Pinsky P, Gatsonis C, et al. Overdiagnosis in low-dose computed tomography screening for lung cancer. JAMA Intern Med 2014; 174: 269-274.

30 Heleno B, Siersma V, Brodersen J. Estimation of overdiagnosis of lung cancer in low-dose computed tomography screening: a secondary analysis of the Danish Lung Cancer Screening Trial. JAMA Intern Med 2018; 178: $1420-1422$.

31 McWilliams A, Tammemagi MC, Mayo JR, et al. Probability of cancer in pulmonary nodules detected on first screening CT. N Engl J Med 2013; 369: 910-919.

32 Yankelevitz DF, Yip R, Smith JP, et al. CT screening for lung cancer: nonsolid nodules in baseline and annual repeat rounds. Radiology 2015; 277: 555-564.

33 Silva M, Prokop M, Jacobs C, et al. Long-term active surveillance of screening detected subsolid nodules is a safe strategy to reduce overtreatment. J Thorac Oncol 2018; 13: 1454-1463.

34 Scholten ET, de Jong PA, de Hoop B, et al. Towards a close computed tomography monitoring approach for screen detected subsolid pulmonary nodules? Eur Resp J 2015; 45: 765-773.

35 Kakinuma R, Noguchi M, Kazuto Ashizawa K, et al. Natural history of pulmonary subsolid nodules: a prospective multicenter study. J Thorac Oncol 2016; 11: 1012-1028.

36 Kobayashi Y, Ambrogio C, Mitsudomi T. Ground-glass nodules of the lung in never-smokers and smokers: clinical and genetic insights. Transl Lung Cancer Res 2018; 7: 487-497.

37 Kondo R, Yoshida K, Kawakami S, et al. Efficacy of CT screening for lung cancer in never-smokers: analysis of Japanese cases detected using a low-dose CT screen. Lung Cancer 2011; 74: 426-432.

38 Liang $\mathrm{F}, \mathrm{Wu} \mathrm{C}, \mathrm{Gu} \mathrm{H}$, et al. Lung cancer incidence in female rises significantly in urban sprawl of Shanghai after introduction of LDCT screening. Lung Cancer 2019; 132: 114-118.

39 Lee HW, Jin KN, Lee JK, et al. Long-term follow-up of ground-glass nodules after 5 years of stability. $J$ Thorac Oncol 2019; 14: 1370-1377.

40 Kim YW, Kang H-R, Kwon BS, et al. Low-dose chest computed tomographic screening and invasive diagnosis of pulmonary nodules for lung cancer in never-smokers. Eur Respir J 2020; 56: 2000177. 